\title{
Rifampicin-Induced Thrombocytopenia: A Case Report and Short Review of the Literature
}

\author{
Authors: \\ Epameinondas Koumpis, Konstantina Papathanasiou, Ioannis \\ Papakonstantinou, Iliana Tassi, Anastasia Serpanou, Eleni Kapsali, \\ *Eleftheria Hatzimichael \\ Department of Haematology, Faculty of Medicine, School of Health Sciences, \\ University of Ioannina, Ioannina, Greece \\ *Correspondence to ehatzim@uoi.gr \\ Disclosure: \\ The authors have declared no conflicts of interest. \\ Received: \\ 31.07.20 \\ Accepted: \\ 05.01.21 \\ Keywords: \\ Drug-induced immune thrombocytopenia (DITP), rifampicin, thrombocytopenia. \\ Citation: \\ EMJ. 2021; DOI/10.33590/emj/20-00193.
}

\section{Abstract}

Thrombocytopenia may be associated with a variety of conditions and risks depending on its severity, ranging from mild epistaxis to life-threating bleeding. Many drugs or herbal remedies can cause thrombocytopenia by either inhibiting platelet production and/or enhancing their destruction from the peripheral blood mediated via an immunological mechanism implicating drug-dependent antibodies. The latter entity is called drug-induced immune thrombocytopenia: a life-threatening, under-recognised condition, which is often a diagnostic challenge. Rifampicin is a widely used, well-tolerated, and effective bactericidal drug. Adverse events, except for gastrointestinal effects, headache, skin rash, and pruritus, are uncommon. The authors herein report on a patient with isolated thrombocytopenia with a recent medical history of brucellosis on rifampicin and doxycycline. Thrombocytopenia was proved to be rifampicin-induced. Also presented is a short review of the literature on this rare subject, which should be of great importance to clinicians.

\section{INTRODUCTION}

Thrombocytopenia is generally defined as a platelet count below the lower normal limit, $<150 \times 10^{9} / \mathrm{L}$, although many suggest that a cutoff value of $100 \times 10^{9} / \mathrm{L}$ is more appropriate to identify clinically significant thrombocytopenia. It may be associated with a variety of conditions and risks ranging from mild epistaxis to lifethreating bleeding. At the time of initial presentation, thrombocytopenia may be isolated or combined with other cytopenias. The cause of thrombocytopenia may be unclear, ${ }^{2}$ and the clinician is usually faced with distinguishing

among various possible pathologies such as sepsis, disseminated intravascular coagulation, microangiopathic processes, or autoimmune disease. ${ }^{3,4}$ Many drugs and components, including nutritional supplements or herbal remedies, ${ }^{5}$ can also cause thrombocytopenia by either inhibiting platelet production and/or enhancing their destruction from the peripheral blood mediated via drug-induced immune thrombocytopenia (DITP). ${ }^{6}$

DITP is a life-threatening, under-recognised condition, and is often a diagnostic challenge. ${ }^{7}$ Detection of drug-dependent antibodies (DDabs) in a patient's serum or plasma with 
suspected DITP is often not possible. This is untrue for heparin-induced-thrombocytopenia, for which the detection of antibodies against platelet factor 4-heparin complex can confirm the suspected diagnosis. Most patients with DITP have multiple comorbidities and other potential causes of thrombocytopenia; ${ }^{7}$ thus, patients with DITP are often diagnosed with idiopathic immune thrombocytopenic purpura. It should be noted that heparin-induced-thrombocytopenia is a special case of drug-induced thrombocytopenia with a mechanism unrelated to other DITP.

Rifampicin is a widely used, well-tolerated, and effective bactericidal drug. It is the most commonly used drug for nontuberculous mycobacterial disease but may also be part of antituberculosis or antibrucellosis regimens, or serve as prophylaxis following exposure to Neisseria meningitidis or Haemophilus influenzae. Adverse events are uncommon, except for gastrointestinal effects, headache, skin rash, and pruritus.

Brucellosis is a zoonotic disease with a wide clinical spectrum. Haematological abnormalities have been seen in brucella-infected patients and the most encountered are anaemia and leukopenia. ${ }^{8}$ Isolated thrombocytopenia has been reported in up to $8 \%$ of cases by Akdeniz et al., ${ }^{9}$ whereas immune-mediated thrombocytopenia has also been reported. ${ }^{9,10}$ The authors of this case report describe a patient with isolated thrombocytopenia with a recent medical history of brucellosis treated with rifampicin and doxycycline. Thrombocytopenia was proved to be rifampicin-induced. They also present a short review of the literature on this rare subject.

\section{CASE PRESENTATION}

A 38-year-old male was referred to the Department of Haematology in the hospital of the authors' place of work for thrombocytopenia. He had a medical history of brucellosis 3 months previously and he had been administered rifampicin $900 \mathrm{mg}$ daily and doxycycline $100 \mathrm{mg}$ twice per day. He had stopped the prescribed treatment before the completion of the recommended period of 6 weeks.

One week before his admission, he was evaluated in another hospital because of malaise, lowgrade fever, and fatigue, symptoms compatible with his previous history of incompletely treated brucellosis. The patient was administered the same antibiotic medication of rifampicin and doxycycline. During his hospitalisation, he presented with ecchymoses on both upper limbs and petechial rash on the oral mucosa. His complete blood count was normal except for low platelets $\left(14 \times 10^{9} / \mathrm{L}\right)$. The patient was started on corticosteroid therapy for a presumed diagnosis of immune thrombocytopenic purpura.

Upon admission, the patient's platelet count was $44 \times 10^{9} / \mathrm{L}$. Corticosteroid therapy was continued while the rest of his medication was stopped. A differential work-up for his thrombocytopenia was initiated. The peripheral blood smear confirmed isolated thrombocytopenia with no morphological abnormalities in the erythroid or myeloid lineage. Prothrombin time and activated partial thromboplastin time were within normal limits. The patient was negative for hepatitis C, HIV, Epstein-Barr virus, cytomegalovirus, and helicobacter pylori (breath test). Antinuclear antibodies were also negative and serum immunoglobulins were within normal range. On the same day, a bone marrow aspiration with cultures was conducted with no abnormal findings. The following day, doxycycline and rifampicin were reintroduced. A critical decrease in the number of platelets was noted from $44 \times 10^{9} / \mathrm{L}$ to $7 \times 10^{9} / \mathrm{L}$. A clinical suspicion of drug-induced thrombocytopenia prompted the discontinuation of rifampicin, which resulted in a rapid increase in the number of platelets. Unfortunately, laboratory tests for DDAbs were not available in the authors' hospital.

The patient showed clinical improvements during his hospitalisation. IgM-specific antibodies for brucella and bone marrow culture were negative and IgG-specific antibodies for brucella were positive. For this reason, discontinuation of doxycycline was also decided after discharge. The patient was discharged after 5 days, having no symptoms and a platelet count of $106 \times 10^{\%} / \mathrm{L}$ (Figure 1). Upon follow-up 1-week postdischarge, the patient's clinical examination was unremarkable, and the platelet count was $315 \times 10^{9} / \mathrm{L}$. The patient was advised to avoid future use of rifampicin. 


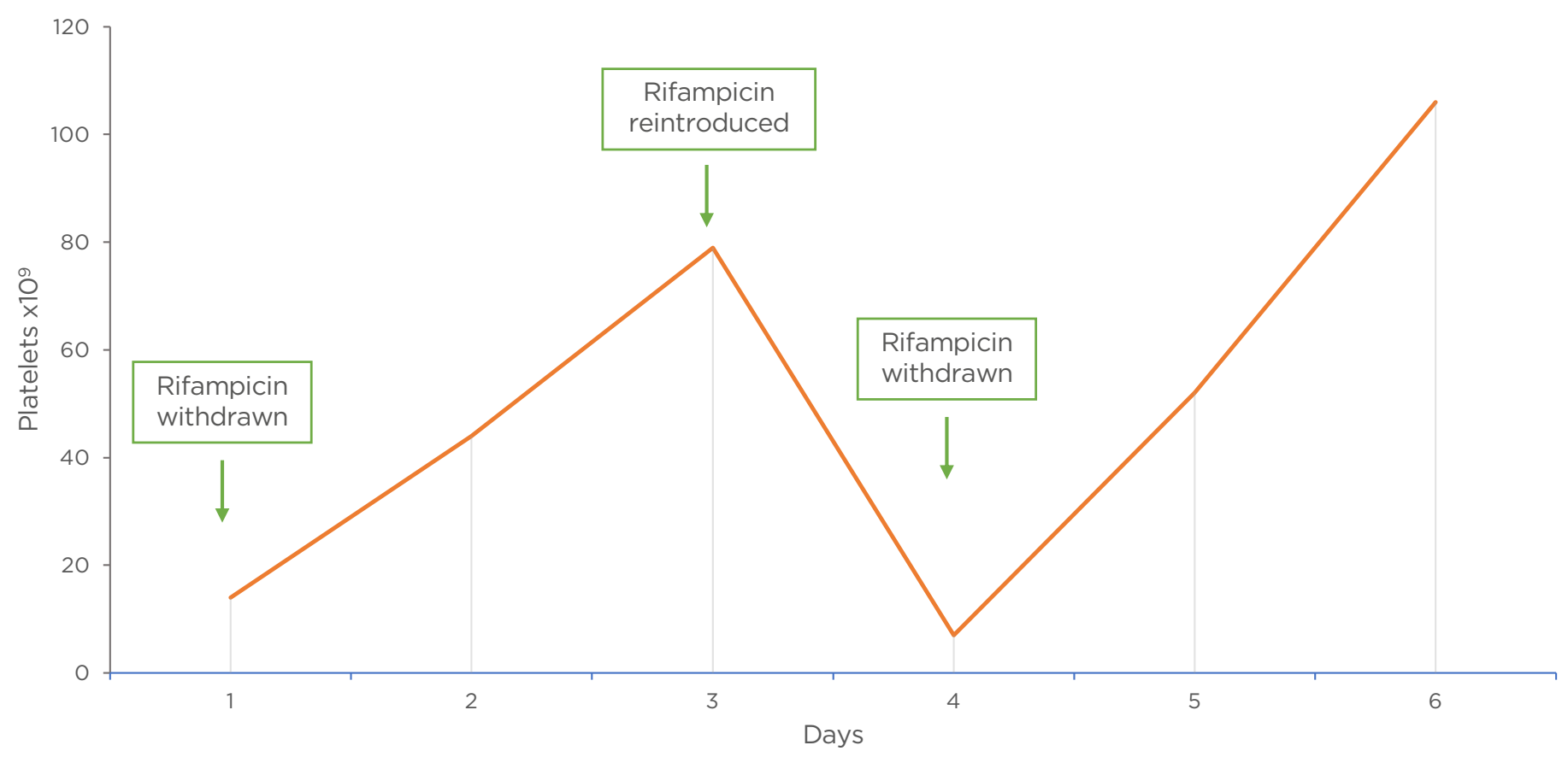

Figure 1: Platelet levels during hospitalisation in a patient with rifampicin-induced thrombocytopenia.

This figure illustrates the levels of platelets (PLT; x109/L) during hospitalisation. Rifampicin was stopped on Day 1 and was resumed on Day $3\left(79 \times 10^{9} / L\right)$. The next day, because of a significant decrease in the platelet count $\left(7 \times 10^{9} / L\right)$, rifampicin was stopped again, and the number of platelets increased rapidly thereafter. Follow-up 1 week postdischarge showed unremarkable clinical examination and platelet count of $315 \times 10^{9} / \mathrm{L}$.

\section{DISCUSSION}

The authors of this review present a patient with rifampicin-induced thrombocytopenia. The considered differential diagnoses in this case were vast: pseudothrombocytopenia was eliminated because there was no platelet aggregation in the peripheral blood smear; myelodysplastic syndrome or aplastic anaemia were excluded after evaluation of the peripheral blood and bone marrow. Thrombotic thrombocytopenic purpura was considered but there were no schistocytes in the peripheral blood smear, there were no symptoms of anaemia, and renal function, bilirubin, and lactate dehydrogenase were normal; disseminated intravascular coagulopathy was eliminated because the clotting screen and fibrinogen were normal. Common variable immunodeficiency was considered but Ig levels were within normal limits; infections were examined and the patient was negative for hepatitis C, HIV, Epstein-Barr virus, cytomegalovirus, and helicobacter pylori. Lastly, autoimmune disease was disregarded because antinuclear antibodies were also negative and the patient did not report arthralgias, sun sensitivity, skin rash, or mouth ulcers.

There are certain criteria that need to be fulfilled to prove a causal relationship between thrombocytopenia and a candidate drug, as suggested by George et al. in 1998.1 These criteria are as follows: 1. Therapy with the candidate drug must precede thrombocytopenia, and recovery from thrombocytopenia must be complete and sustainable with the discontinuation of the drug; 2. The candidate drug must be the only one used before the onset of thrombocytopenia, or other drugs must be continued or reintroduced after discontinuation of therapy with the candidate drug with a sustained normal platelet count; 3. Other causes should be excluded; 4. Re-exposure to the candidate drug results in recurrent thrombocytopenia."

In this present case, rifampicin was a candidate drug causing thrombocytopenia 
and the discontinuation of the drug led to the resolution of thrombocytopenia. Other causes of thrombocytopenia were excluded, such as normal bone marrow, negative screening for viruses, and autoimmune diseases, and reintroduction of the candidate drug caused a recurrent decrease of platelets. In addition, the proposal of thrombocytopenia caused by other drugs was excluded, because doxycycline was continued when the treatment of rifampicin was stopped and there were no other drugs administered. This patient, therefore, fulfilled all four criteria required, leading to a definitive level of evidence (Level I) that thrombocytopenia was drug-induced. The Adverse Drug Reaction Probability Scale, developed by Naranjo et al.,12 was used with a score of 9 (Definite).

Limited epidemiologic data exist for DITP. An estimated incidence of approximately 10 cases per 1,000,000 in the population per year has been reported,,$^{13}$ although this is likely to be an underestimate. DITP can be caused by two different mechanisms: suppression of platelet production (myelosuppressive drugs and drugs that affect the metabolism of folic acid) and platelet destruction. Destruction of platelets can be induced by at least three different mechanisms, which include the hapten-dependent antibodies, the platelet-reactive autoantibodies, the DDabs, or both drug-dependent and drug-specific antibodies. ${ }^{14,15}$ Hapten-dependent antibodies bind covalently to the protein of the thrombocyte membrane and this complex induces immunemediated cell destruction. The second mechanism encompasses antibody binding against the platelet membrane, while the third mechanism of DDabs is not well understood..$^{14,15}$ Bougie et al. ${ }^{15}$ proposed a model in which the administration of the drug increased the affinity between DDabs and the epitope on a platelet glycoprotein.

DITP induced by DDabs is classically caused by antibodies inducing platelet destruction by the reticuloendothelial system only in the presence of the drug. ${ }^{16}$ Quinine was the first drug recognised as being involved in this group of DITP; however 'quinine-type' antibodies typically recognise a restricted set of epitopes on glycoprotein $\mathrm{lb} / \mathrm{IX}^{17}$ and glycoprotein $\mathrm{llb} /$ Illa complexes of the platelet membrane. ${ }^{18}$ Rifampicin-induced thrombocytopenia has been linked to the presence of antiplatelet antibodies, which have higher avidity for platelet membrane in the presence of rifampicin. ${ }^{19}$ The epitope of rifampicin-dependent antibodies is on glycoprotein IX, a subunit of the glycoprotein IX complex. ${ }^{20}$ An experimental study showed that rifampicin-dependent antibodies also targeted glycoprotein Ilb/IIla. ${ }^{21} \quad$ Currently available laboratory tests can identify the causative agent in patients for whom there is suspicion and clinical evidence of DITP, including rifampicin-induced thrombocytopenia. ${ }^{22,23}$ However, the aetiology of DITP can be established when a prompt rise in platelets occurs with the discontinuation of the drug, while its reintroduction leads to a new drop in platelet count, as noted in this patient.

Rifampicin-induced thrombocytopenia was first described by Blajchman et al. ${ }^{24}$ in 1970. Since then, almost 40 cases have been documented in the literature and a recent review by Cooper and Ghanima $^{3}$ included rifampicin in the group of common drugs that cause immune-mediated thrombocytopenia. ${ }^{3}$ Case reports and series of rifampicin-induced thrombocytopenia are presented in Table 1.

Rifampicin-induced thrombocytopenia usually occurs when the drug is either intermittently administered or reintroduced after a voluntary discontinuation, as is the case in this present study. ${ }^{25,26}$ Bansal et al. ${ }^{27}$ described a case of rifampicin-induced thrombocytopenia after reexposure to rifampicin 10 years later. It has been suggested that drug sensitivity and induction of thrombocytopenia remains indefinitely. ${ }^{57}$ In four cases in the literature, thrombocytopenia occurred with no previous exposure to rifampicin, ${ }^{28-31}$ whereas in three more, reported by Lee et al., ${ }^{32}$ thrombocytopenia occurred following a 4-month period of rifampicin prophylaxis in a tuberculosis outbreak. ${ }^{32}$

Corticosteroids have been used in cases where thrombocytopenia was the result of an immune-mediated mechanism; their benefit is not proven. ${ }^{58}$ Transfusion of platelets is indicated when a patient presents with severe thrombocytopenia or wet purpura owing to a great risk of intracranial haemorrhage. . $^{14,33}$ Plasmapheresis and intravenous Ig have been used in drug-induced thrombocytopenia.59,60 All treatment options are controversial. The most crucial step is the immediate discontinuation of the drug. 
Table 1: Cases of rifampicin-induced thrombocytopenia in literature.

\begin{tabular}{|c|c|c|}
\hline Reference & Sample size $(n)$ & Study design \\
\hline Mehta et al., ${ }^{19}$ (1996) & 3 & Case report \\
\hline Agrawal et al.,22 (2012) & 1 & Case report \\
\hline Blajchman et al., ${ }^{24}$ (1970) & 1 & Case report \\
\hline Verma et al., ${ }^{25}$ (2010) & 1 & Case report \\
\hline Kindelán et al., ${ }^{26}$ (1994) & 1 & Case report \\
\hline Bansal et al., ${ }^{27}$ (2013) & 1 & Case report \\
\hline Bashir et al.,28 (2016) & 1 & Case report \\
\hline Lee et al., ${ }^{29}$ (1989) & 2 & Case report \\
\hline Pau et al., ${ }^{30}$ (1987) & 1 & Case report \\
\hline Esposito et al., ${ }^{31}$ (1971) & 1 & Case report \\
\hline Lee et al., ${ }^{32}(2012)$ & 87 (who received rifampicin) & Cohort study $(n=3.4 \%)$ \\
\hline Kang et al., ${ }^{33}$ (2010) & 1 & Case report \\
\hline Nair et al., ${ }^{34}$ (2009) & 1 & Case report \\
\hline Mauricio et al., ${ }^{35}$ (2019) & 1 & Case report \\
\hline Arnold et al., ${ }^{36}$ (2015) & $2,500^{*}$ & Observation study \\
\hline Yakar et al.,37 (2013) & 1 & Case report \\
\hline Dixit et al., ${ }^{38}$ (2012) & 1 & Case report \\
\hline Mori et al., ${ }^{39}$ (2011) & 1 & Case report \\
\hline Davis et al., ${ }^{40}$ (2009) & $6^{+}$ & Case report \\
\hline Kant et al., ${ }^{41}$ (2008) & 1 & Case report \\
\hline Muñoz et al.. ${ }^{42}$ (2008) & 1 & Case report \\
\hline Scognamiglio et al., ${ }^{43}$ (2008) & 2 (1 received rifampicin) & Case report \\
\hline Garg et al., ${ }^{44}$ (2007) & 1 & Case report \\
\hline Krivoy et al., ${ }^{45}$ (2001) & 1 & Case report \\
\hline Pereira et al.. ${ }^{46}$ (2000) & 1 & Case report \\
\hline Tricerri et al., ${ }^{47}$ (1997) & 1 & Case report \\
\hline Martínez et al., ${ }^{48}$ (1994) & 1 & Case report \\
\hline Juang et al.,49 (1992) & 1 & Case report \\
\hline Bhasin et al.,50 (1991) & 1 & Case report \\
\hline Burnette et al., ${ }^{51}$ (1989) & 1 & Case report \\
\hline Kakaiya et al.,52 (1989) & 1 & Case report \\
\hline Hadfield ${ }^{53}(1980)$ & 1 & Case report \\
\hline Poole et al., 54 (1971) & $49 \ddagger$ & Clinical trial \\
\hline Ferguson $^{55}$ (1971) & 1 & Case report \\
\hline Leggat $^{56}$ (1971) & 1 & Case report \\
\hline
\end{tabular}

*Thrombocytopenia (121/1,000 infant days)

†Thrombocytopenia $(n=1)$

¥Thrombocytopenia $(n=3)$ 
The reintroduction of the drug is not indicated even if small doses are to be used;25 however, Nair et al. ${ }^{34}$ supported the continuation of rifampicin along with treatment for immune thrombocytopenia.

\section{CONCLUSION}

Although thrombocytopenia is a rare side effect of rifampicin, healthcare professionals should be aware of its life-threatening risks. Thrombocytopenia can be complicated with mild bleeding events such as epistaxis or bruising but also with severe events such as gastrointestinal bleeding and acute subdural haemorrhage. ${ }^{33}$ Notably, previous exposure to the drug is not necessary for the presentation of thrombocytopenia. Patients should be advised to avoid future use of the drug in order to prevent the potential detrimental effects of thrombocytopenia.

\section{References}

1. Smock KJ, Perkins SL. Thrombocytopenia: an update. Int J Lab Hematol. 2014;36(3):269-78.

2. Arnold DM, Lim W. A rational approach to the diagnosis and management of thrombocytopenia in the hospitalized patient. Semin Hematol. 2011;48(4):251-8.

3. Cooper N, Ghanima W. Immune thrombocytopenia. N Engl J Med. 2019;381(10):945-55.

4. Rousan TA et al. Recurrent acute thrombocytopenia in the hospitalized patient: sepsis, DIC, HIT, or antibioticinduced thrombocytopenia. Am J Hematol. 2010;85(1):71-4.

5. Ohmori T et al. Acute thrombocytopenia induced by jui, a traditional herbal medi-cine. J Thromb Haemost. 2004;2(8):1479-80.

6. Aster $\mathrm{RH}$ et al. Drug-induced immune thrombocytopenia: pathogenesis, diagnosis, and management. J Thromb Haemost. 2009;7(6):911-8.

7. Arnold DM et al. Approach to the diagnosis and management of druginduced immune thrombocytopenia. Transfus Med Rev. 2013;27(3):137-45.

8. al-Eissa $Y$, al-Nasser $M$. Haematological manifestations of childhood brucellosis. Infection. 1993;21(1):23-6.

9. Akdeniz $\mathrm{H}$ et al. Hematological manifestations in brucellosis cases in Turkey. Acta Med Okayama. 1998;52(1):63-5.

10. Gurkan E et al. Immune thrombocytopenic purpura associated with Brucella and Toxoplasma infections. Am J Hematol. 2003;74(1):52-4

11. George JN et al. Drug-induced thrombocytopenia: a systematic review of published case reports. Ann Intern Med. 1998;129(11):886-90.

12. Naranjo CA et al. A method for estimating the probability of adverse drug reactions. Clin Pharmacol Ther. 1981;30(2):239-45.
13. Chong $\mathrm{BH}$ et al. Drug-induced immune thrombocytopenia. Hematol Oncol Clin North Am. 2013;27(3):52140.

14. van den Bemt PMLA et al. Druginduced immune thrombocytopenia. Drug Saf. 2004;27(15):1243-52.

15. Bougie DW et al. Patients with quinine-induced immune thrombocytopenia have both "drugdependent" and "drug-specific" antibodies. Blood. 2006;108(3):922-7.

16. Vayne $\mathrm{C}$ et al. Pathophysiology and diagnosis of drug-induced immune thrombocytopenia. J Clin Med. 2020;9(7):2212

17. Burgess JK et al. Quinine-dependent antibodies bind a restricted set of epitopes on the glycoprotein Ib-IX complex: characterization of the epitopes. Blood. 1998;92(7):2366-73

18. Peterson JA et al. Fine specificity of drug-dependent antibodies reactive with a restricted domain of platelet GPIIIA. Blood. 2008;111(3):1234-9.

19. Mehta YS et al. Rifampicin-induced immune thrombocytopenia. Tuber Lung Dis. 1996;77(6):558-62.

20. Burgess JK et al. Rifampicindependent antibodies bind a similar or identical epitope to glycoprotein IX-specific quinine-dependent antibodies. Blood. 2000;95(6):1988 92.

21. Sun P et al. Rifampicin-dependent antibodies target glycoprotein llb/ IIla and cause clearance of human platelets in NOD/SCID mice. Br Haematol. 2016;172(1):137-40.

22. Agrawal A et al. Do not miss rifampicin-induced thrombocytopenic purpura. BMJ Case Rep. 2012;2012:bcr1220115282.

23. Arnold DM et al. A systematic evaluation of laboratory testing for drug-induced immune thrombocytopenia. J Thromb Haemost. 2013;11(1):169-76.

24. Blajchman MA et al. Rifampicininduced immune thrombocytopenia. Br Med J. 1970;3(5713):24-6.
25. Verma AK et al. Rifampicin-induced thrombocytopenia. Indian J Pharmacol. 2010;42(4):240-2

26. Kindelán JM et al. Rifampin-induced severe thrombocytopenia in a patient with pulmonary tuberculosis. Ann Pharmacother. 1994;28(11):1304-5.

27. Bansal R et al. A case of thrombocytopenia caused by rifampicin and pyrazinamide. Indian J Pharmacol. 2013;45(4):405-7.

28. Bashir $\mathrm{H}$ et al. Rifampcin-induced thrombocytopaenia purpura. Indian J Chest Dis Allied Sci. 2016;58(3):18990.

29. Lee CH, Lee CJ. Thrombocytopenia--a rare but potentially serious side effect of ini-tial daily and interrupted use of rifampicin. Chest. 1989;96(1):202-3.

30. Pau AK, Fisher MA. Severe thrombocytopenia associated with once-daily rifampin therapy. Drug Intell Clin Pharm. 1987;21(11):882-4

31. Esposito R, Vitali D. Rifampicin and thrombocytopenia. Lancet. 1971;2(7722):491-2.

32. Lee $\mathrm{SH}$ et al. Adverse events and development of tuberculosis after 4 months of rifampicin prophylaxis in a tuberculosis outbreak. Epidemiol Infect. 2012;140(6):1028-35.

33. Kang SY et al. Acute subdural hemorrhage associated with rifampicin-induced thrombocytopenia. Neurol Sci. 2010;31(2):199-200.

34. Nair S, Carroll WD. Carry on doctor. Arch Dis Child. 2009;94(9):734-5.

35. Maurício J et al. Severe rifampicininduced thrombocytopenia in a patient with miliary tuberculosis. Pulmonology. 2019;26(4):247-9.

36. Arnold CJ et al. Rifampin use and safety in hospitalized infants. Am J Perinatol. 2015;32(6):565-70.

37. Yakar F et al. Isoniazidand rifampicin-induced thrombocytopenia. Multidiscip Respir Med. 2013;8(1):13. 
38. Dixit R et al. Thrombocytopenia due to rifampicin. Lung India. 2012;29(1):90-2.

39. Mori M et al. A case of acute and severe thrombocytopenia due to readministration of rifampicin. J Infect Chemother. 2011;17(2):288-90.

40. Davis LC et al. Meningococcal disease in a backpackers hostel in Scotland: a risk assessment for prophylaxis. Euro Surveill. 2009;14(31):19291.

41. Kant S et al. Rifampicin, ethambutol and pyrazinamide-induced thrombocytopenia. Int J Clin Pharmacol Ther. 2008;46(8):440-2.

42. Muñoz ME et al. Rifampin-related acute renal failure, thrombocytopenia, and leuko-cytoclastic vasculitis. Ann Pharmacother. 2008;42(5):727-8.

43. Scognamiglio $\mathrm{F}$ et al. Flow cytometry in the diagnosis of druginduced thrombocytopenia: two illustrative cases. Am J Hematol. 2008:83(4):326-9.

44. Garg R et al. Rifampicin induced thrombocytopenia. Indian J Tuberc. 2007:54(2):94-6.

45. Krivoy $\mathrm{N}$ et al. Rifampin-induced thrombocytopenia: diagnosis by a novel in vitro lymphocyte toxicity assay. Isr Med Assoc J. 2001;3(7):536-
46. Pereira J et al. Glycoprotein Ib/IX complex is the target in rifampicininduced immune thrombocytopenia. $\mathrm{Br}$ J Haematol. 2000;110(4):907-10.

47. Tricerri $A$ et al. Adverse drug reaction to rifampin: a case with long lasting antiplatelet antibodies. Panminerva Med. 1997;39(1):64-6.

48. Martínez E et al. Evidence implicating rifampin-independent antiplatelet antibodies in the pathogenesis of rifampin-induced immune thrombocytopenia. Clin Infect Dis. 1994;19(2):351-3.

49. Juang $Y C$ et al. Acute renal failure and severe thrombocytopenia induced by rifampicin: report of a case. J Formos Med Assoc. 1992;91(4):475-6.

50. Bhasin DK et al. Can rifampicin be restarted in patients with rifampicininduced thrombocytopenia? Tubercle. 1991;72(4):306-7.

51. Burnette PK et al. Rifampinassociated thrombocytopenia secondary to poor compliance. DICP. 1989;23(5):382-4.

52. Kakaiya RM et al. Rifampin-induced immune thrombocytopenia. A case report. Vox Sang. 1989;57(3):185-7.

53. Hadfield JW. Rifampicin-induced thrombocytopenia. Postgrad Med J. 1980;56(651):59-60.
54. Poole $\mathrm{G}$ et al. Potentially serious side-effects of high-dose twiceweekly rifampicin. Postgrad Med J. 1971;47(553):727-47.

55. Ferguson GC. Rifampicin and thrombocytopenia. Br Med J. 1971:3(5775):638.

56. Leggat PO. Rifampicin and thrombocytopenia. Lancet. 1971;2(7715):103-4.

57. Aster $\mathrm{RH}$ et al. Drug-induced immune thrombocytopenia. N Engl J Med. 2007;357(6):580-7.

58. Pedersen-Bjergaard $U$ et al. Druginduced thrombocytopenia: clinical data on 309 cases and the effect of corticosteroid therapy. Eur J Clin Pharmacol. 1997;52(3):183-9.

59. Ray JB et al. Intravenous immune globulin for the treatment of presumed quinidine-induced thrombocytopenia. DICP. 1990;24(78):693-5.

60. Pourrat O. Treatment of drug-related diseases by plasma exchanges. Ann Med Interne (Paris). 1994;145(5):35760. 